\title{
ATLAS Inner Detector performance
}

\author{
Szymon Gadomski ${ }^{1}$ \\ CERN and INP Cracow
}

\begin{abstract}
The ATLAS Inner Detector consists of three subsystems using different tracking detector technologies: silicon pixels, silicon strips and straw tubes. The combination gives ATLAS a robust, hermetic and efficient tracking system, able to reconstruct tracks at the highest foreseen LHC luminosities. The inner detector provides vertex and momentum measurements, electron identification and some $K / \pi$ separation. The beam pipe design of ATLAS was changed recently, causing an increase of the radius of the first tracking layer. The consequences are discussed in this paper.
\end{abstract}

Talk presented at BEAUTY 2000, Ma'agan, Israel, 17.09.2000

\footnotetext{
${ }^{1}$ Szymon.Gadomski@cern.ch
} 


\section{Introduction}

The ATLAS inner detector and its performance are described in detail in $[1,2,3,4]$. This paper contains a summary, focusing on aspects relevant to B-physics and on recent changes. The ATLAS trigger for B-physics, based in a large part on the inner detector performance, is described separately in these proceedings [5].

The layout of the inner detector and the basic properties of its subsystems are described in section 2 . The performance of the complete system is discussed in section 3 and the conclusions are presented in section 4 .

\section{ID Subsystems}

The inner detector will be installed inside a solenoid magnet producing a $2 \mathrm{~T}$ magnetic field. The available space is a cylinder with $110 \mathrm{~cm}$ radius and $7 \mathrm{~m}$ total length. Three detector subsystems, using different technologies, occupy that space. From the inside out, the inner detector consists of:

- a silicon Pixel detector,

- the Semiconductor Tracker (SCT) using silicon microstrips,

- the Transition Radiation Tracker (TRT) using straw tubes.

The layout is shown schematically in Fig.1.

The beam pipe design of ATLAS has been changed this year, a double wall was introduced. Two $0.8 \mathrm{~mm}$ thick layers of beryllia are separated by a $4 \mathrm{~mm}$ vacuum gap. The new design is motivated by the need to bake out the beam pipe while the first pixel layer, called the B-layer, will already be installed. The change in outer diameter of the beam pipe is from $50 \mathrm{~mm}$ to $69.2 \mathrm{~mm}$. As a consequence the mean radius of the sensors in the first layer has increased from 43 to $50.5 \mathrm{~mm}$. From the performance point of view this change of layout is the most significant one in recent years. The consequences are discussed in the next section. Other recent layout changes, related to the routing of services, are less significant.

The pixel detector has two more barrels beyond the B-layer, named layer "1" and "2". The average radii are 10.1 and $13.2 \mathrm{~cm}$ respectively. Coverage in the forward direction is assured by 5 disks on each side, at $z=49$ to $77 \mathrm{~cm}$ and $R=10$ to $19 \mathrm{~cm}$. The B-layer is removable and can be replaced during the lifetime of the experiment. The other layers and the disks are in principle 
not replaceable and will need to stand 10 years of the LHC operation. The central value ${ }^{2}$ of the current radiation damage predictions indicates that such a scenario is indeed possible and even includes a safety margin, particularly if oxygenated detectors are used. The pixel modules can operate at $600 \mathrm{~V}$ bias voltage and can function when the detectors are only partially depleted.

The pixel detector consists of 2146 modules, the total surface of silicon is $2.87 \mathrm{~m}^{2}$ (counting only detectors) and the number of channels is about $10^{8}$. The pixel size is $50 \times 300 \mu \mathrm{m}^{2}$ in the B-layer, $50 \times 400 \mu \mathrm{m}^{2}$ elsewhere, with all modules identical except in the B-layer. The modules are about $2.5 \times 6.5 \mathrm{~cm}^{2}$. The total power of $\sim 17 \mathrm{~kW}$ needs to be absorbed by an evaporative cooling system using $\mathrm{C}_{3} \mathrm{~F}_{8}$ as the medium.

The electronics has data compression, adjustable offset at every channel and provides pulse height information using time over threshold (ToT). The ToT was one of the options of the pixel readout, it has recently been approved as a baseline choice. The resolution per point benefits from the use of pulse height information, by which an interpolation of the track position between two pixels is made possible. A recent beam test demonstrates the use of this technique [7], the results are summarised in Fig.2.

Both the approval of the ToT as a baseline choice and the pixel beam test results using the ToT are recent. The resolutions that were assumed for all detector performance and physics studies done until now were "binary" - with no interpolation between the pixels. The "binary" resolutions are $\sim 12 \mu \mathrm{m}$ in $R \phi$ and 69 to $77 \mu \mathrm{m}$ in $z$ (barrel) or $R$ (forward disks) [3]. All the performance results reported in section 3 , as well as all the physics projections presented in $[8,9]$ and in earlier publications, were obtained using the "binary" resolutions.

The occupancy in the pixel detector is highest in the B-layer. Because of the fine granularity the occupancy is of the order of $10^{-4}$ in the B-layer at $L=10^{34} \mathrm{~cm}^{-2} \mathrm{~s}^{-1}$. As the readout is organised in column pairs, the occupancy per column pair is a relevant figure as well. That occupancy is $10 \%$ on average at $L=10^{34} \mathrm{~cm}^{-2} \mathrm{~s}^{-1}$ in the B-layer. The occupancy distribution has a long tail, meaning that the occupancy can reach much larger values causing readout inefficiency. The inefficiency due to high local occupancies is estimated to be below 1\%. The exact value depends on the final implementation of the electronics, which does not yet exist.

\footnotetext{
${ }^{2}$ It is perhaps worth bearing in mind that the predictions for the doses to which the detectors will be exposed have a $30 \%$ uncertainty [6]. There are also other uncertainties in radiation damage predictions, often difficult to quantify, for instance related to dose rate effects.
} 
The SCT has 4 cylinders at radii of 30 to $52 \mathrm{~cm}$ in the barrel part. The total length of the barrel is $\sim 1.5 \mathrm{~m}$. There are 2112 identical barrel modules, their total detector area is $34 \mathrm{~m}^{2}$. The forward SCT has 9 disks, 1976 modules and $27 \mathrm{~m}^{2}$ of silicon detectors. There are three types of forward modules, depending on the radius. The total number of SCT channels is $6.3 \times 10^{6}$ and the total power is $\sim 40 \mathrm{~kW}$. The cooling system is similar to that of the pixel detector.

Most SCT modules ${ }^{3}$ have four silicon strip detectors, two on either side. The sides are rotated by $40 \mathrm{mrad}$ with respect to each other in order to provide a measurement of the dimension along the strips - $z$ in the barrel and $R$ in the forward disks. The strip pitch is $80 \mu \mathrm{m}$ in the barrel modules and the approximate dimensions of the barrel module are $6.5 \times 13 \mathrm{~cm}^{2}$. The forward modules have a key-stone shape, with strips that are not parallel but radial. The pitch varies from 63 to $85 \mu \mathrm{m}$ at module centres. The SCT electronics is purely binary and provides no pulse height information beyond the $0 / 1$ answer with respect to a threshold. The threshold is common for each 128 channel chip, but the offsets can be corrected on a channel by channel basis. The $R \phi$ resolution of the SCT is around $22 \mu \mathrm{m}$ in the barrel and on average $24 \mu \mathrm{m}$ in the forward. The expected occupancy of the first barrel layer is $\sim 6 \times 10^{-3}$ at $L=10^{34} \mathrm{~cm}^{-2} \mathrm{~s}^{-1}$.

The TRT is built using straws of $4 \mathrm{~mm}$ diameter. The gas mixture filling the straws is $70 \% \mathrm{Xe}, 20 \% \mathrm{CF}_{4}, 10 \% \mathrm{CO}_{2}$. The barrel part extends from 56 to $107 \mathrm{~cm}$ in radius. The $\sim 50000$ barrel straws are laid along the $z$ direction and their length is $2 \times 74 \mathrm{~cm}$, with readout of the separated halves at both ends. The $\sim 320000$ straws in the end-cap TRT disks are radial, extending from $R=64$ to $103 \mathrm{~cm}$ (from 48 to 103 in the last wheels).

The straws are surrounded by materials in which the electrons will produce transition radiation (TR). The TR photons will then be absorbed and produce large signals on the straws. The TRT readout electronics will have two thresholds, a lower threshold for detecting signal from ionisation in gas and a higher threshold for detecting the TR. The electronics will also give the ToT for the lower threshold. That additional information has been shown to help particle identification (see next section). The occupancy of the TRT at $L=10^{34} \mathrm{~cm}^{-2} \mathrm{~s}^{-1}$ can reach almost $40 \%$ in the innermost parts, but it does not exceed 10 to $15 \%$ in the rest of the system.

The TRT electronics will also measure drift time in order to improve the spatial resolution. This measurement is affected if the straw is crossed by two

\footnotetext{
${ }^{3}$ The innermost modules on all the forward disks have only one detector on either side.
} 
tracks. The $R \phi$ resolution varies from $170 \mu \mathrm{m}$ to $240 \mu \mathrm{m}$, depending on local occupancy.

\section{Combined performance}

The inner detector provides hermetic coverage up to $\eta=2.5$. There are at least eleven precision points on each track, three from pixels and eight from the SCT, maintained at all $\eta$ values. The average number of TRT points is 35 per track over the whole rapidity coverage. The number of points drops in the crack region between barrel and forward (the worst value is 23 points at $\eta=0.9)$ but it recovers to above 36 at larger $\eta$ values.

The pattern recognition is robust. Three reconstruction algorithms, each starting from a different subdetector, give comparable results. The efficiency for finding muons is $98 \%$ at all $p_{T}$ values above $1 \mathrm{GeV} / c$ and at all $\eta$ values up to $\eta=2.5$. The efficiency for finding pions is $\sim 90$ to $95 \%$ at $p_{T}>1 \mathrm{GeV} / c$, dependent on momentum and rapidity, limited by the interaction of pions with detector material. There is little dependence of the reconstruction efficiency on pile-up of events. The only place in which the track finding starts to lose efficiency and to reconstruct tracks wrongly is in jets of several hundred GeV, which are not of particular interest from the B-physics point of view. Some selected track finding efficiencies are illustrated in Fig.3.

The efficiency for reconstructing $K_{S}^{0} \rightarrow \pi^{+} \pi^{-}$decays is $41 \%$ in the $B^{0} \rightarrow$ $J / \Psi K_{S}^{0}$ events passing ATLAS selection criteria. This total $K_{S}^{0}$ reconstruction efficiency breaks down into two components. The efficiency for passing a set of fiducial cuts is $70 \%$. For decays in the fiducial region the efficiency for reconstructing both pions, fitting them into a common vertex and for accepting the reconstructed mass is the remaining $59 \%$.

The impact parameter resolution has suffered recently because of an increase of the B-layer radius. Other less important factors were an increase of the pixel module material and the thicker beam pipe. The resolutions before and after the changes are shown in Fig.4.

The ATLAS physics projections described in these proceedings [8, 9], as well as in earlier publications, have not yet been updated with the latest vertex resolutions. There exists one estimate of the impact of the changes on physics performance - a deterioration of 15 to $20 \%$ is expected for the resolution on the $B_{S}^{0}$ proper decay time. This would mean a 15 to $20 \%$ deterioration of the sensitivity range for the $\Delta m_{S}$.

The current impact parameter resolution of ATLAS can be parameterised 
in the following way:

$$
\begin{aligned}
\sigma\left(d_{0}\right) & =12 \oplus \frac{88}{p_{T} \sqrt{\sin \Theta}} & {[\mu \mathrm{m}] } \\
\sigma\left(z_{0}\right) & =95 \oplus \frac{160}{p_{T} \sqrt{\sin ^{3} \Theta}} & {[\mu \mathrm{m}] }
\end{aligned}
$$

where $d_{0}$ is the transverse impact parameter, $z_{0}$ is the $z$ of the point of closest approach of the track to the beam line and $p_{T}$ is in $\mathrm{GeV} / c$. The use of ToT in pixels will likely reduce the asymptotic term of the impact parameter resolutions, but a proper study was not carried out yet.

The momentum resolution of the inner detector can be parameterised as

$$
\sigma\left(1 / p_{T}\right)=0.00036 \oplus \frac{0.013}{p_{T} \sqrt{\sin \Theta}} \quad\left[\mathrm{GeV}^{-1}\right]
$$

To give a relevant example, for the decays of $B^{0} \rightarrow \pi^{+} \pi^{-}$triggered and reconstructed by ATLAS the mass resolution is $\sigma\left(m_{B^{0}}\right)=70 \mathrm{MeV}$.

The TRT can identify electrons by using the TR effect, counting high threshold hits on the track. A complementary technique, using the $d E / d x$ measurement available via the ToT information (see previous section) was developed and tried in beam test recently [10]. The combination gives ATLAS

a good ability to identify electrons. Pion rejection factor at $90 \%$ electron efficiency is shown in Fig.5. The $d E / d x$ information can also be used for $K / \pi$ separation, although the separation is not better than $1 \sigma$ (see Fig.6).

\section{Conclusions}

The recent enlargement of the beam pipe has caused an increase of the radius of the first tracking layer by $17 \%$. This causes a slight deterioration of the impact parameter resolutions. It is estimated that the $B_{S}^{0}$ proper time resolution and the $\Delta m_{S}$ sensitivity range are degraded by 15 to $20 \%$. Other consequences for ATLAS B-physics remain to be studied.

Beam test data analyses have brought new and encouraging results for the TRT and for the pixels. In TRT the particle identification is enhanced, while in pixels an improved spatial resolution was observed. In both cases the progress was made by using time-over-threshold technique.

The inner detector gives hermetic tracking up to $\eta=2.5$, robust pattern recognition, precise vertex measurement, electron identification and even some $K / \pi$ separation. 


\section{References}

[1] ATLAS Collaboration, Inner Detector Technical Design Report, Vol. I, CERN/LHCC/97-16 (1997).

[2] ATLAS Collaboration, Inner Detector Technical Design Report, Vol. II, CERN/LHCC/97-17 (1997).

[3] ATLAS Collaboration, Pixel Detector Technical Design Report, CERN/LHCC/98-13 (1998).

[4] ATLAS Collaboration, Detector and Physics Performance Technical Design Report, CERN/LHCC/99-14 (1999).

[5] John Baines, ATLAS trigger for B-physics, these proceedings.

[6] Shaun Roe, CERN, private communication.

[7] Tommaso Lari, a presentation available from Clara Troncon, http://cern.ch/troncon/testbeam.html.

[8] David Rousseau, ATLAS Overview and physics projections, these proceedings.

[9] Simon Robins, ATLAS B-production measurements, these proceedings.

[10] T. Akesson et al., Particle Identification using the Time-over-Threshold Method in the ATLAS Transition Radiation Tracker, ATLAS internal note ATL-INDET-2000-021, also submitted to Nuclear Instruments and Methods. 


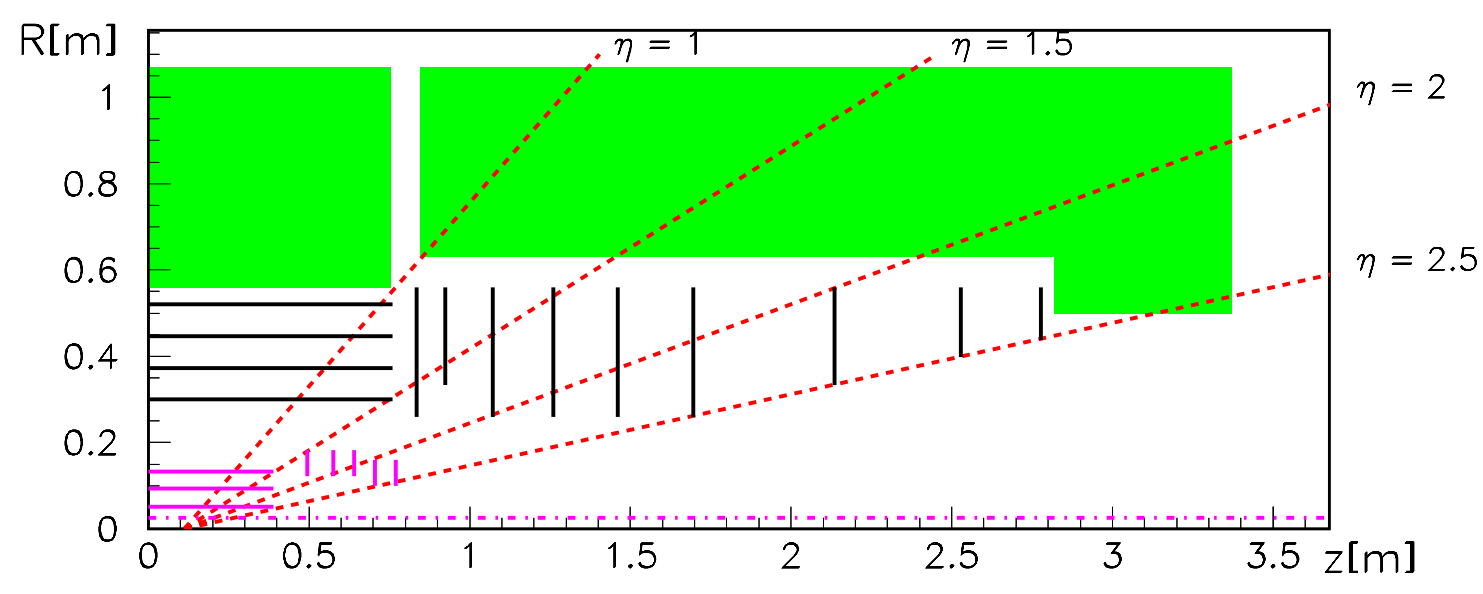

Figure 1: A schematic cross-section of the ATLAS inner detector. The TRT is shown as a grey box, the SCT with black lines and the pixels with grey lines. 


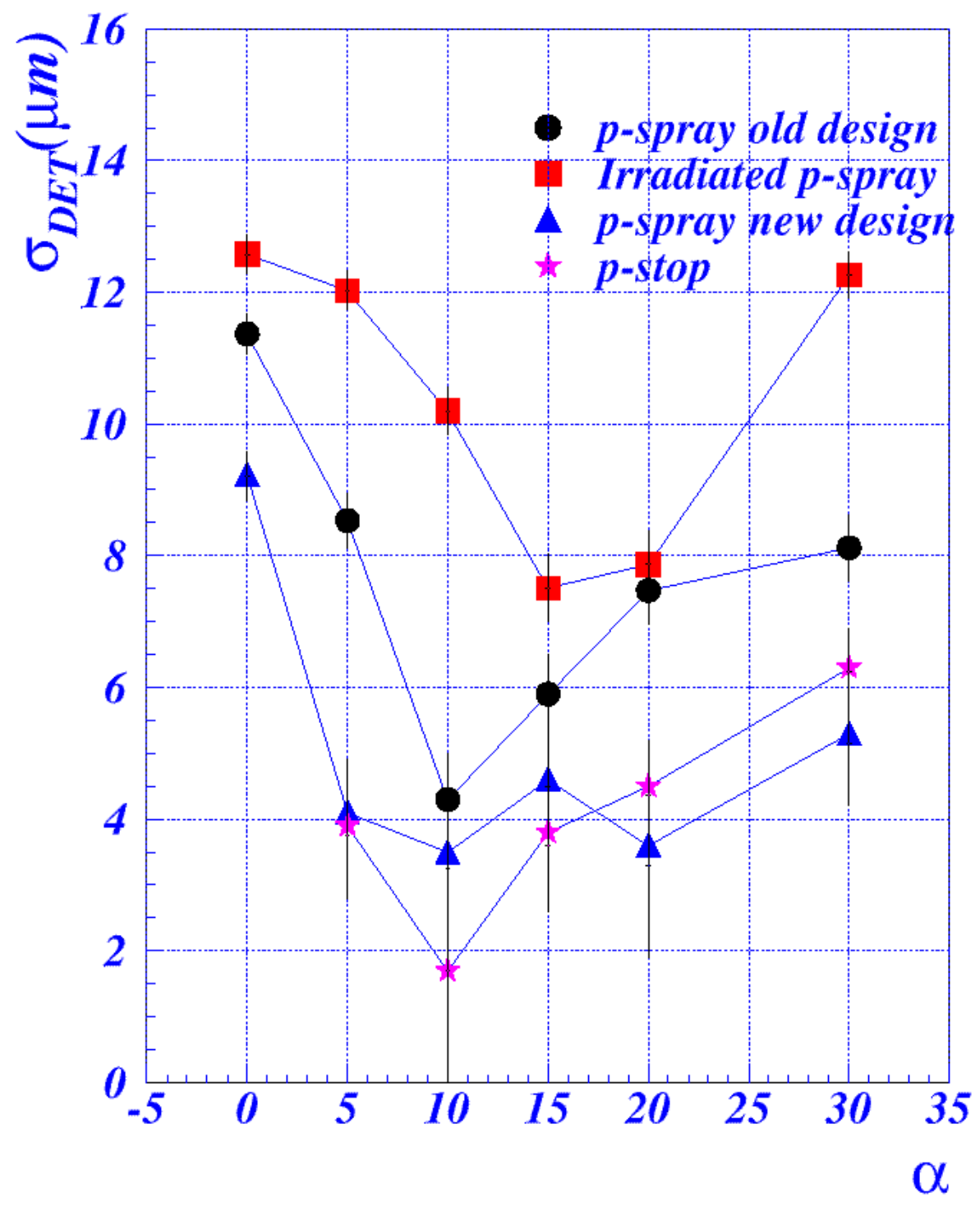

Figure 2: The $R \phi$ resolution per track point in the pixel module, shown as a function of track incidence angle, measured in a beam test with various detector designs. 

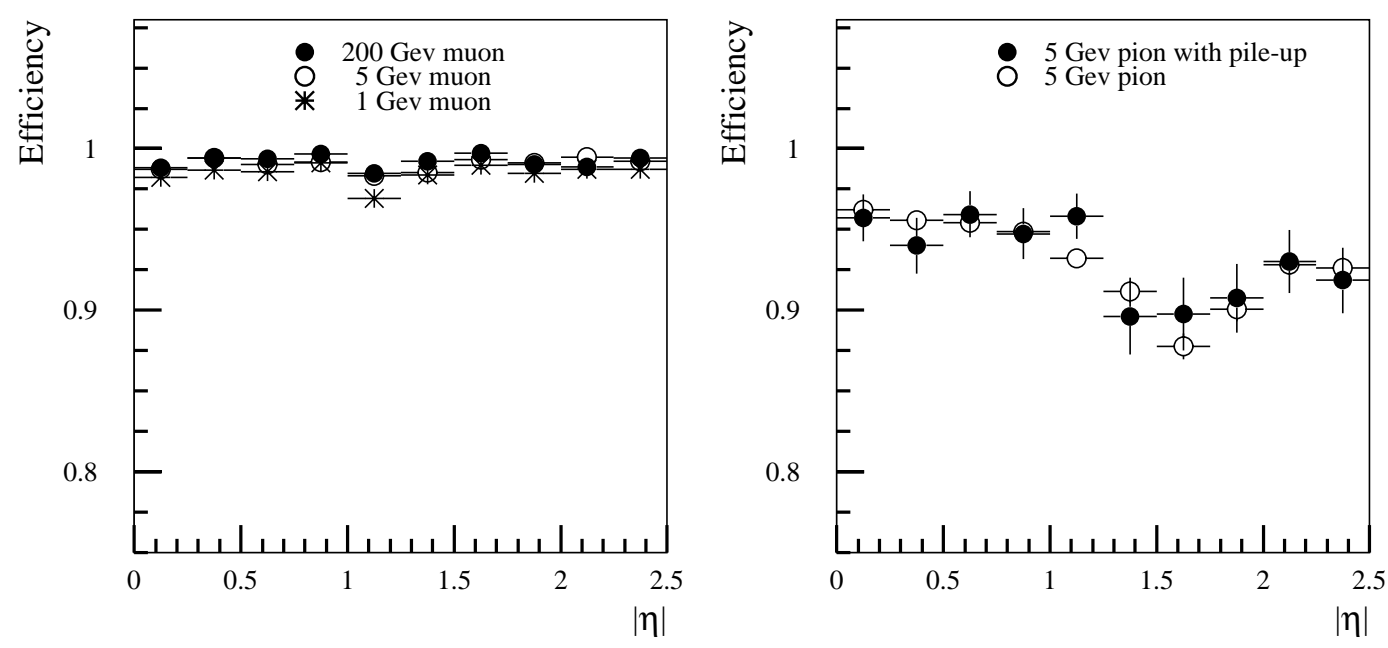

Figure 3: Track finding efficiency for muons of different momenta (left) as well as for pions at $p_{T}=5 \mathrm{GeV} / c$ with and without pileup (right). 

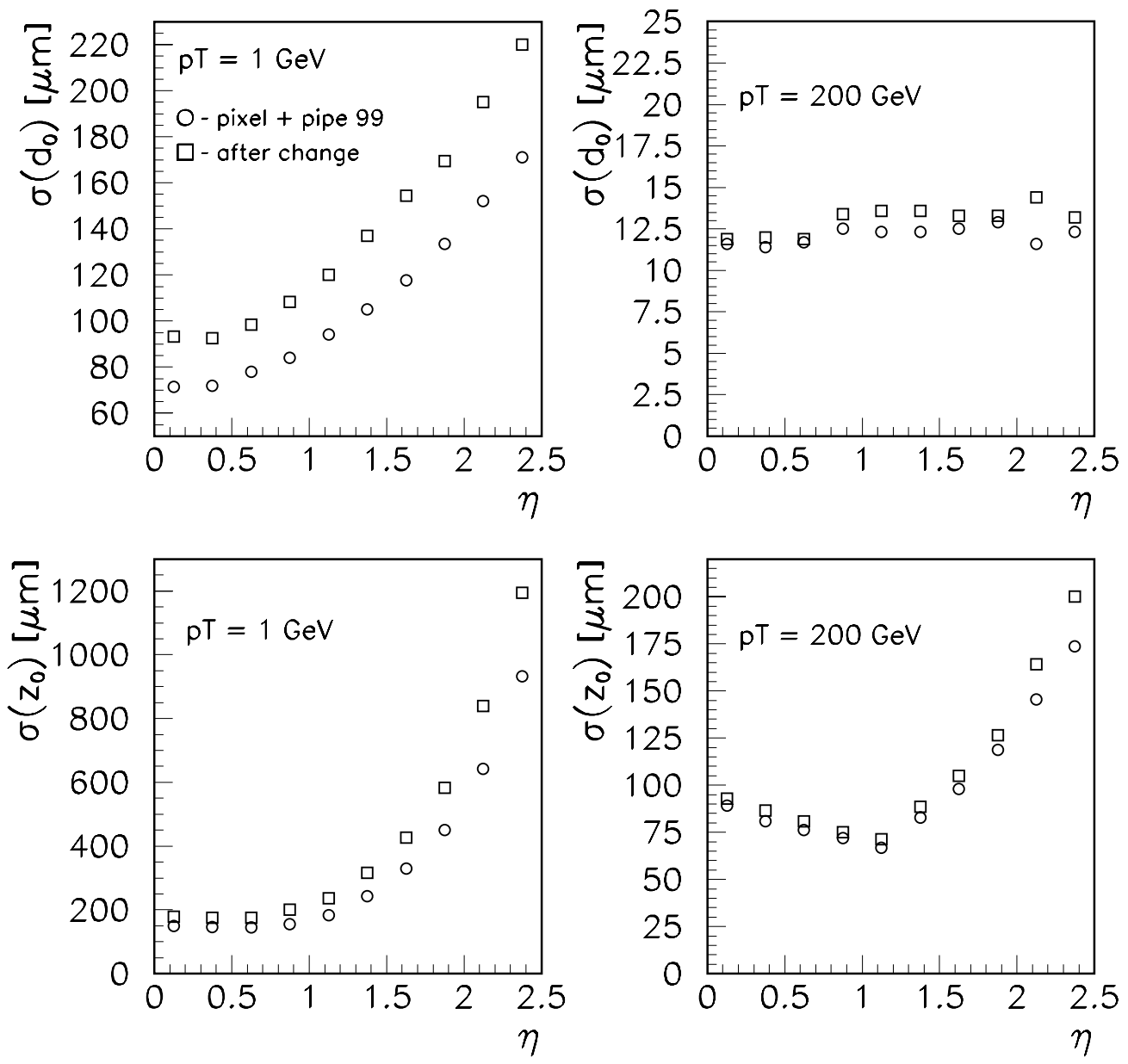

Figure 4: Impact parameter resolutions of the inner detector before and after the latest change of the beam pipe and of the pixel detector layout. The resolutions in the transverse impact parameters $d_{0}$ and in the " $\mathrm{z}$ " of the closest approach of the track to beam line $z_{0}$ are shown as a function of $\eta$ for track $p_{T}$ of 1 and $200 \mathrm{GeV} / c$. 


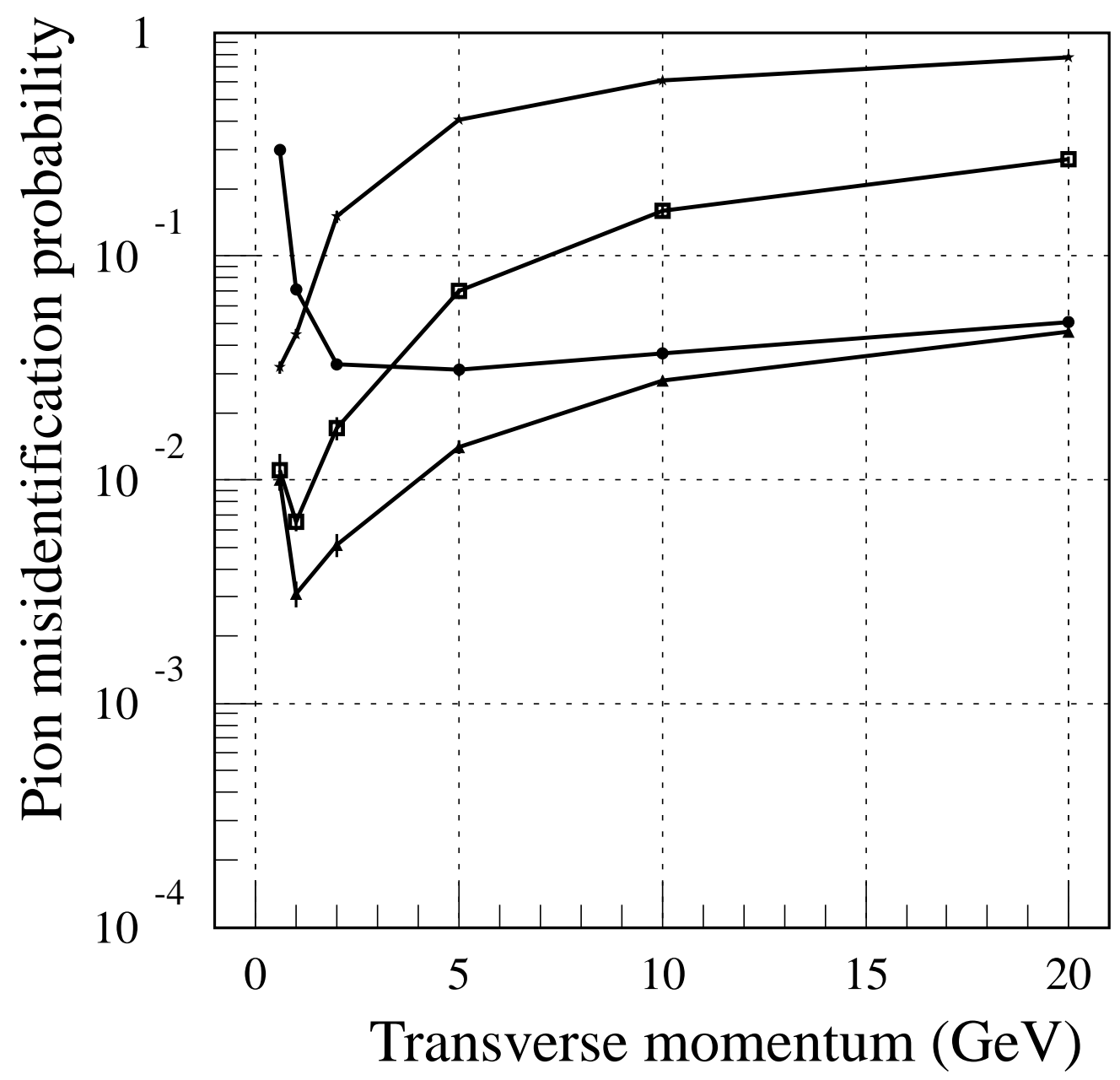

Figure 5: Pion misidentification probability as a function of transverse momentum at $90 \%$ electron efficiency for single tracks at $\eta=0.3$ and without pile-up. The performance is shown for $d E / d x$ using only high-threshold hits (stars), for $d E / d x$ using all hits (squares), for transition-radiation cluster counting technique (circles), and for the overall combined method (triangles). 


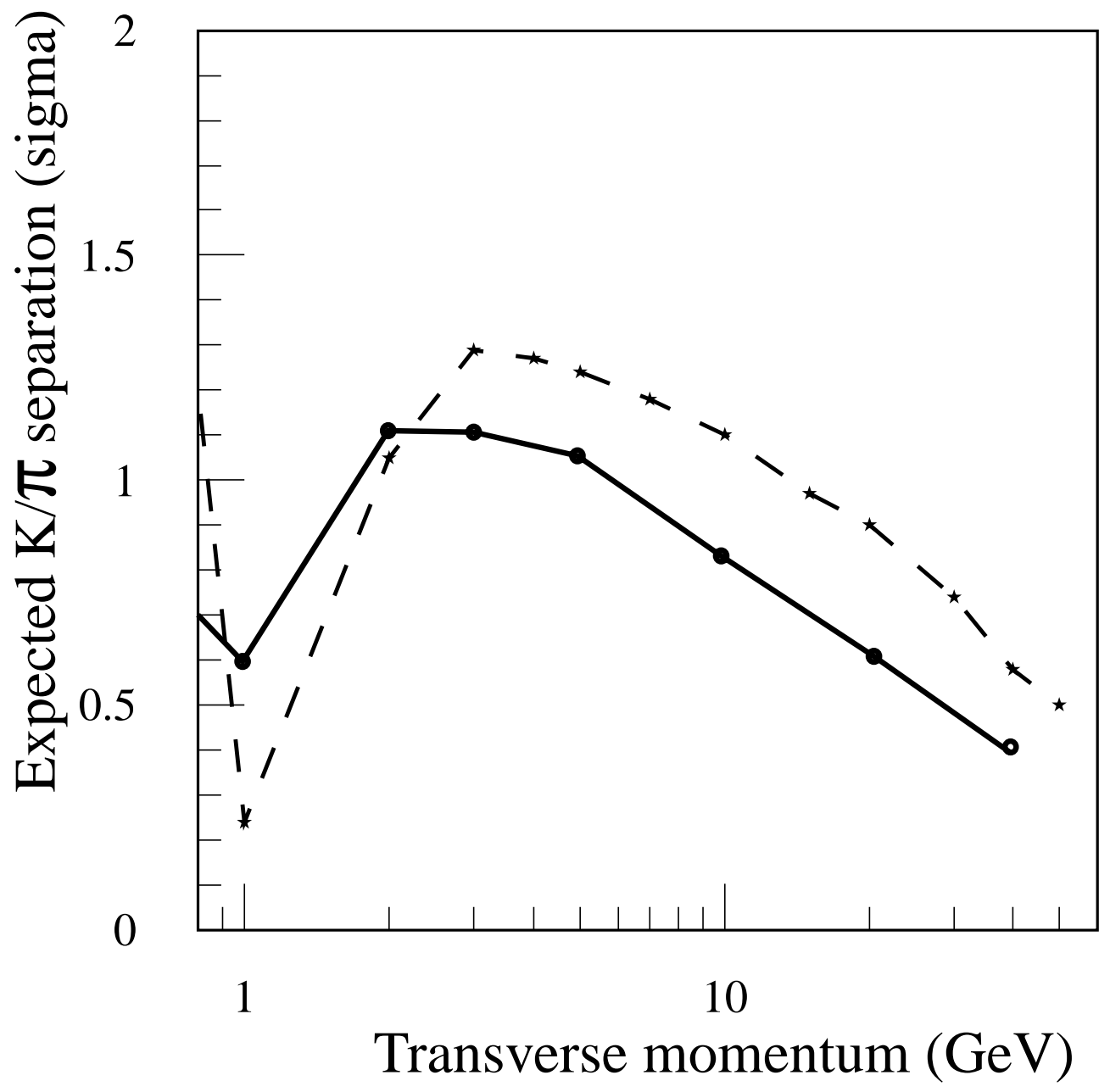

Figure 6: Expected $\mathrm{K} / \pi$ separation as a function of transverse momentum (no pile-up effects included). The separation is shown as an average over the full rapidity coverage (solid line) and at $\eta=0.3$ (dotted line). 